\title{
Properties of melt processed chitosan and aliphatic polyester blends
}

\author{
V.M. Correlo, L.F. Boesel, M. Bhattacharya*, J.F. Mano, N.M. Neves, R.L. Reis \\ 3B's Research Group_Biomaterials, Biodegradables and Biomimetics, Department of Polymer Engineering, \\ University of Minho, Campus de Gualtar, Braga 4710-057, Portugal
}

Accepted 18 April 2005

\begin{abstract}
Chitosan was melt blended with poly- $\varepsilon$-caprolactone (PCL), poly(butylene succinate) (PBS), poly(lactic acid) (PLA), poly(butylene terephthalate adipate) (PBTA), and poly(butylene succinate adipate) (PBSA). For the chitosan/PBS blend, the amount of chitosan was varied from $25 \%$ to $70 \%$ by weight. The remaining polyesters had $50 \%$ of chitosan by weight. Addition of chitosan to PBS or PBSA tends to depress the melting temperature of the polyester. The crystallinity of the polyesters (PCL, PBS, PBSA) containing 50\% chitosan decreased. Adding chitosan to the blends decreased the tensile strength but increased the tensile modulus. Chitosan displayed intermediate adhesion to the polyester matrix. Microscopic results indicate that the skin layer is polyester rich, while the core is a blend of chitosan and polyester. Fractured surface of chitosan blended with a high $T_{\mathrm{g}}$ polymer, such as PLA, displayed a brittle fracture. Blends of chitosan with PCL, PBTA, or PBSA display fibrous appearances at the fractured surface due to the stretching of the polymer threads. Increasing the amount of chitosan in the blends also reduced the ductility of the fractured surface. The chitosan phase agglomerated into spherical domains or were clustered into sheaths. Pull-out of chitosan particles is evident in tensile-fractured surfaces for blends of chitosan with ductile polymers but absent in the blends with PLA. PBS displays a less lamellar orientation when compared to PCL or PBSA. The orientation of the polyesters (PCL, PBSA) does not seem to be affected by the addition of chitosan. The two main diffraction peaks observed using WAXS are unaffected by the addition of chitosan.
\end{abstract}

(C) 2005 Elsevier B.V. All rights reserved.

Keywords: Melt processing; Chitosan; Aliphatic polyesters; Properties

\section{Introduction}

A number of natural polymers are being employed as biomaterials. Naturally occurring polymers include collagen, chitin, silk, alginate, starch, and elastin. Natural polymers offer the advantage of being similar to macromolecular substances, which the biological environment is prepared to recognize and to deal with metabolically. Problems associated with the stimulation of chronic inflammatory reaction and toxicity by synthetic polymers are largely suppressed or eliminated by using natural polymers. On the other hand, natural polymers are frequently quite immunogenic. Another attractive characteristic of natural polymers is their ability

\footnotetext{
* Corresponding author at: Department of Biosystems and Agricultural Engineering, University of Minnesota, 1390 Eckles Avenue, St. Paul, MN 55108, USA. Tel.: +1 612625 7733; fax: +1 1626243005 .

E-mail address: bhatt002@umn.edu (M. Bhattacharya).
}

to be degraded by naturally occurring enzymes, implying that the implant will be degraded and eventually metabolized by physiological mechanisms. Thus, useful properties can be obtained by blending natural and synthetic polymers.

Synthetic polymers used in the biomaterials field include biodegradable aliphatic polyesters, such as polyhydroxybutyrate and its copolymer polyhydroxybutyrate-co-valerate, poly(lactic acid), poly(glycolic acid), poly- $\varepsilon$-caprolactone, and their copolymers. The ester bonds in these synthetic polymers hydrolyze to nontoxic natural metabolites and are eliminated from the body via respiration [1]. Synthetic biodegradable polymers used as biomaterials range in tensile strength from 16 to $50 \mathrm{MPa}$ and modulus from 400 to $3000 \mathrm{MPa}[2]$.

Chitin is a biopolymer found in nature and is mainly derived from the exoskeletons of crustaceans, insects and mollusks, and the cell walls of microorganisms [3]. Like 
cellulose, chitin is a glucose-based, unbranched polysaccharide. However, it differs from cellulose at the $\mathrm{C}_{2}$ carbon, where instead of a hydroxyl group, chitin has an acetamide residue. Chitosan ( $\alpha(1 \rightarrow 4)$-2-amino-2-deoxy- $\beta$-D-glucan $)$, a mucopolysaccharide, is the alkaline deactylated product of chitin and has structural similarities to glycosaminoglycans, thus, mimicking their functional behavior. Chitosan is reported to be non-toxic, biodegradable, and biocompatible [4,5].

Solution blending of chitin and chitosan with synthetic polymers has been studied. Graft copolymerization of chitosan with acrylonitrile, methylmethacrylate (MMA), methacrylic acid, 2-hydroxyethylmethacrylate (HEMA), and acryl-amide has been reported in the literature. Similarly, styrene, vinyl acetate, acrylamide, MMA, and HEMA, have also been grafted on chitosan. Grafting of chitosan with $N, N^{\prime}-$ dimethylaminoethylmethacrylate (DMAEMA) has also been reported [6-10]. The tensile strength, crystallinity, and degree of swelling of grafted chitosan films decreased with increased levels of grafting with synthetic polymers. The grafted films showed improved thermal stability. Functional groups have been grafted on chitosan powder to increase its solubility [11]. Blends of polyurethane-chitosan were prepared using the solvent casting method [12], and the mechanical properties were evaluated. Poor phase properties were reported. Hybrid materials of chitosan with polynosic (viscose rayon) were generated by a mechanical blending method. Chitin and chitosan were reacted with 1,6-diisocyanatohexane (poly-urea\{urethanes\}) in DMA- $\mathrm{LiCl}$ solutions and their properties evaluated [13]. Biodegradable films of chitosan containing polyethylene glycol (PEG) or polyvinyl alcohol (PVA) were prepared by mixing PEG or PVA with a solution of chitosan acetate, and films were prepared by the casting method $[2,14]$. Homogenous films with increased value of initial temperature of thermal degradation were produced.

Little research has been conducted in the melt blending of synthetic polyester and chitosan. The objective of this research was to evaluate the properties of chitosan and biodegradable aliphatic polyesters (poly- $\varepsilon$-caprolactone (PCL), poly(butylene succinate) (PBS), poly(lactic acid) (PLA), poly(butylene terephthalate adipate) (PBTA), and poly(butylene succinate adipate) (PBSA). The tensile properties (tensile strength and tensile modulus) and thermal properties of the blends were evaluated. The amount of chitosan in the blends containing PBS was varied at $25 \%, 50 \%$, and $70 \%$. For the remaining polyester, the chitosan content was at $50 \%$. The tensile and thermal properties were evaluated. The morphology of fractured and etched surfaces was evaluated using scanning electron microscopy (SEM). Optical microscopy was used to distinguish the stained chitosan phase in the blend. Small angle X-ray scattering (SAXS) and wide angle X-ray scattering (WAXS) were used to study the effect of processing on both the lamellar and crystalline structure of these blends.

\section{Experimental methods}

\subsection{Materials}

The polyesters used include poly- $\varepsilon$-caprolactone (PCL), poly(butylene succinate) (PBS), poly(lactic acid) (PLA), poly(butylene terephthalate adipate) (PBTA), and poly(butylene succinate adipate) (PBSA). The chitosan used had a degree of deacetylation of approximately $85 \%$. Polycaprolactone resins PCL 787 (MFI 4), commercially available as $\mathrm{TONE}^{\mathrm{TM}}$ polymer, were obtained from Union Carbide Chemicals and Plastics Division, Bound Brook, New Jersey. Eastar Bio Copolyester $14766^{\mathrm{TM}}$, a butanediol, adipate, and terephthalate copolymer (MFI $\sim 20$ ), was obtained from Eastman Chemical Company, Kingsport, Tennessee. Bionolle ${ }^{\mathrm{TM}} 1050$, a polybutylene succinate copolymer (MFI 50), and Bionolle ${ }^{\mathrm{TM}} 3001$, a poly(butylene succinate adipate) copolymer (MFI $\sim 1.0$ ) were obtained from Showa Highpolymer Co. Ltd., Tokyo, Japan.

\subsection{Processing}

The chitosan/polyester blends containing less than $70 \%$ chitosan were compounded in a counter rotating twin-screw extruder (Carvex, Lisbon, Portugal). The only exception to this was when blends of chitosan ( $50 \%$ by weight) were compounded with B3001. Higher chitosan content pushed the torque in the counter rotating extruder close to the maximum. Alternately, throughput had to be reduced to keep the torque below the upper threshold. Hence, blends containing $70 \%$ chitosan were compounded in a co-rotating twin-screw extruder [Leistriz LSM 36] to improve mixing. Since the torque was close to the maximum limit of the machine, this blend (70\% chitosan $/ 30 \%$ PBS) was processed with the die removed and with added water/glycerol to enhance plasticization. The various blend compositions studied and processing conditions used are summarized in Table 1. The extruded strands were ground using a Coloritron grinder using $5 \mathrm{~mm}$ diameter pellets. The blends were injection molded using an ENGEL injection molding machine to produce tensile test bars. The tensile bars had a neck cross-section area of $2 \mathrm{~mm} \times 4 \mathrm{~mm}$ and a neck length of $20 \mathrm{~mm}$. The conditions used for molding are summarized in Table 2 . The mold was held at a constant temperature of $22 \mathrm{C}$ for all compositions except for PLA where the mold temperature was raised to $30 \mathrm{C}$.

\subsection{Mechanical properties}

The tensile properties were determined using a Universal tensile testing machine (Instron 4505 Universal Machine). Tensile force was taken as the maximum force in the forcedeformation curve. Tensile modulus was estimated from the initial slope of the stress-strain curve. Samples were conditioned at room temperature for at least $48 \mathrm{~h}$ before testing. A crosshead speed of $5 \mathrm{~mm} \mathrm{~min}^{-1}$ was used up to a deformation 
Table 1

Processing conditions used for various blend and composite compositions studied

\begin{tabular}{|c|c|c|c|c|}
\hline \multirow[t]{2}{*}{ Material } & \multicolumn{4}{|l|}{ Processing conditions } \\
\hline & Temperature profile $\left({ }^{\circ} \mathrm{C}\right)$ & Screw speed (rpm) & Die temperature $\left({ }^{\circ} \mathrm{C}\right)$ & Type of machine used \\
\hline $25 \mathrm{C} / 75 \mathrm{PBS}$ & $160 / 160 / 160$ & 15 & 160 & Counter rotating (CARVEX) \\
\hline $50 \mathrm{C} / 50 \mathrm{PBS}$ & $160 / 160 / 160$ & 15 & 160 & Counter rotating (CARVEX) \\
\hline 50C/50 PBTA & $160 / 160 / 160$ & 15 & 160 & Counter rotating (CARVEX) \\
\hline $50 \mathrm{C} / 50 \mathrm{PCL}$ & $160 / 160 / 160$ & 15 & 160 & Counter rotating (CARVEX) \\
\hline $50 \mathrm{C} / 50 \mathrm{PBSA}$ & 160 in all zones & 100 & 160 & Co-rotating (LEISTRITZ) \\
\hline $50 \mathrm{C} / 50 \mathrm{PLA}$ & 175 in first four zones, 180 rest of the zones & 100 & 180 & Co-rotating (LEISTRITZ) \\
\hline $70 \mathrm{C} / 30 \mathrm{PBS}$ & $70 / 90$ and 160 in the rest of the zones & 100 & - & Co-rotating (LEISTRITZ) \\
\hline
\end{tabular}

of $1.5 \%$ after which the speed increased to $50 \mathrm{~mm} \mathrm{~min}^{-1}$. The values reported were the average of at least five specimens.

\subsection{Differential scanning calorimetry}

The DSC experiments were performed in a Perkin-Elmer DSC7 apparatus, using a water-cooling accessory and nitrogen as a purge gas (flux gas of ca. $20 \mathrm{~cm}^{3} \mathrm{~min}^{-1}$ ). Both temperature and heat flux were calibrated with Indium (99.99999\% purity) at a scanning rate of $20^{\circ} \mathrm{C} \mathrm{min}^{-1}$. The samples were obtained by cutting a small piece of material (with ca. $10 \mathrm{mg}$ weight) in the central region of the injection parts. An effort was made to maintain the geometry of the different samples, in order to keep the same thermal resistance. All the experiments were performed at $20{ }^{\circ} \mathrm{C} \mathrm{min}^{-1}$, starting from room temperature. Only the first run was analyzed, which reflects not only the materials contained in the samples, but also the general morphology developed during processing.

\subsection{Optical microscopy}

To analyze the chitosan distribution on the polymer matrix, tensile bars of each blend were stained with a $0.10(\mathrm{w} / \mathrm{v})$ Eosin solution for $10 \mathrm{~min}$ at $32 \pm 0.5^{\circ} \mathrm{C}$. Stained samples were examined by light microscopy (Olympus BH-2) in reflection mode. Photographs of the stained surface were obtained using a microscope digital camera Olympus DP11. Images of

Table 2

Injection molding processing conditions for blends

\begin{tabular}{llcl}
\hline Material & $\begin{array}{l}\text { Injection speed } \\
\left(\mathrm{mm} \mathrm{s}^{-1}\right)\end{array}$ & $\begin{array}{l}\text { Holding } \\
\text { pressure }(\text { bar })\end{array}$ & $\begin{array}{l}\text { Barrel } \\
\text { temperature }\left({ }^{\circ} \mathrm{C}\right)\end{array}$ \\
\hline PBS & 30 & 50 & $90-120-120-140$ \\
PBSA & 30 & 120 & $80-100-120-130$ \\
PBTA & 30 & 50 & $90-120-120-140$ \\
PCL & 80 & 80 & $70-90-100-110$ \\
PLA & 30 & 30 & $155-170-180-190$ \\
25C/75 PBS & 25 & 20 & $110-130-150-160$ \\
50C/50 PBS & 30 & 80 & $110-130-150-160$ \\
50C/50 PBTA & 20 & 30 & $110-130-150-160$ \\
50C/50 PCL & 40 & 20 & $110-130-150-160$ \\
50C/50 PBSA & 65 & 100 & $110-130-150-160$ \\
50C/50 PLA & 70 & 25 & $150-155-155-160$ \\
70C/30 PBS & 80 & 50 & $110-130-150-160$ \\
\hline
\end{tabular}

the cross-section were obtained by cryogenically fracturing the tensile bar. The fractured surface was smoothened using a glass knife for optical clarity.

\subsection{Scanning electron microscopy}

The morphological characterization of the blends was made using a Leica-Cambridge S-360 scanning electron microscope. All the samples were sputter-coated with gold. Several different analyses of the cross-section of the tensile bars were made. These include (i) observations after the tensile tests; (ii) observations after immersing the samples in liquid nitrogen for $1 \mathrm{~min}$ and fracturing the sample in the testing zone to analyze the brittle fracture; and (iii) observations after cryogenically-fractured surfaces were etched with $1 \%$ acetic acid for $3 \mathrm{~h}$ or 1 day. The objective was to dissolve the chitosan since not enough phase contrast between the filler and polyester was obtained in the SEM images. In addition, the extrudates were also observed under SEM.

\subsection{Wide angle (WAXS) and small angle (SAXS) X-ray scattering}

WAXS and SAXS experiments were performed using Xray synchrotron radiation (transmission mode) at the Soft Condensed Matter A2 beamline at HASYLAB (DESY) synchrotron facility in Hamburg (Germany). The experimental setup includes a MARCCD detector for acquiring twodimensional SAXS patterns (sample-to-detector distance being $280 \mathrm{~cm}$ ) and a linear detector for 1D WAXS measurements (distance $23 \mathrm{~cm}$ ). $\mathrm{Cu} \mathrm{K} \alpha$ radiation, with a wavelength of $\lambda=0.154 \mathrm{~nm}$, was employed for both SAXS and WAXS measurements. The injection moulded samples were fixed vertically and the patterns were acquired at room temperature.

\section{Results and discussion}

\subsection{Thermal properties}

The normalized DSC scans of PLA-based materials are shown in Fig. 1a. The injected pure PLA exhibits a glass transition at $62.0^{\circ} \mathrm{C}$ (midpoint), with $\Delta C_{p}\left(T_{\mathrm{g}}\right)=0.305 \mathrm{~J} \mathrm{~g}^{-1}$. The endothermic peak at $65^{\circ} \mathrm{C}$ is associated with structural 

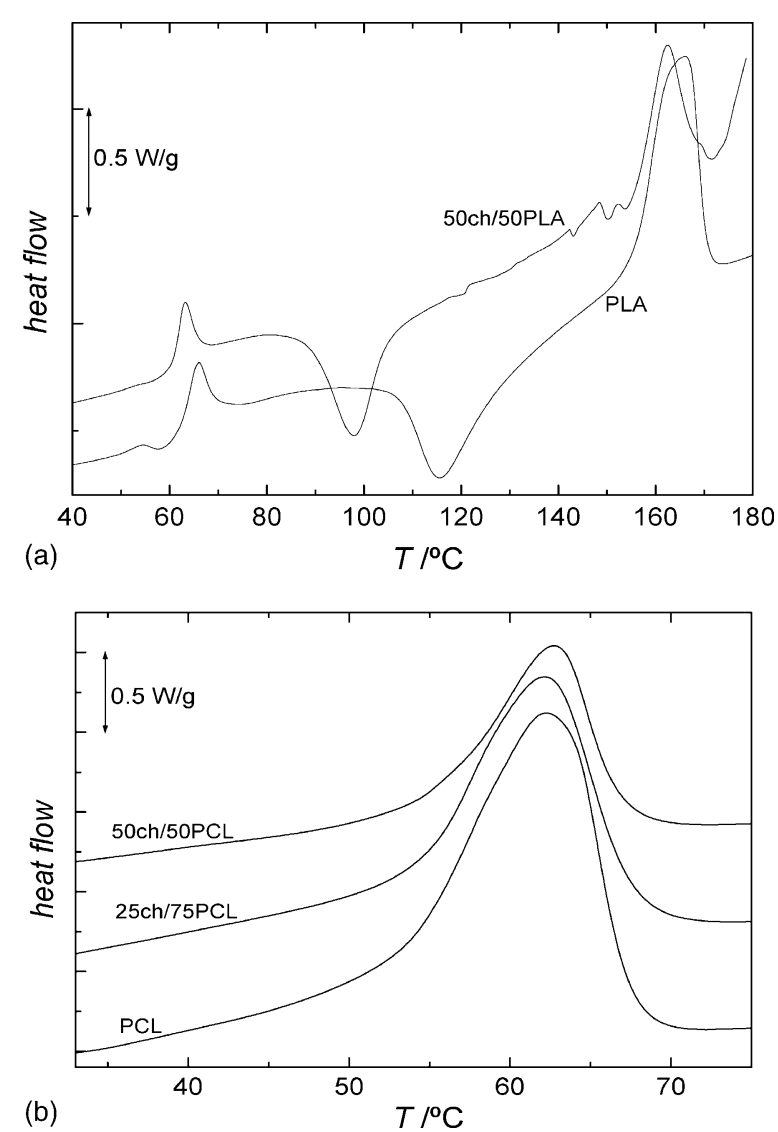

Fig. 1. Representative DSC thermograms, obtained at $20^{\circ} \mathrm{C} \mathrm{min}^{-1}$ of (a) both PLA and 50ch/50PLA materials and (b) PCL-based materials.

relaxation. In fact, the amorphous fraction of the material is in its glassy state at room temperature. Hence, in this non-equilibrium condition, the enthalpy will slowly relax towards its equilibrium values. At higher temperatures, the cold-crystallization is observed at $T_{\mathrm{c}}=107.3^{\circ} \mathrm{C}$ and the melting at $T_{\mathrm{m}}=155.3^{\circ} \mathrm{C}$. The integration of the heat flux curve comprising both processes (between 99 and $174{ }^{\circ} \mathrm{C}$ ) gave $6.5 \mathrm{~J} \mathrm{~g}^{-1}$. This small value indicates that the material is essentially amorphous. This was confirmed by wide-angle X-ray scattering (data not shown).

The blend of PLA with $50 \mathrm{wt} \%$ chitosan has as slightly lower $T_{\mathrm{g}}\left(60.1^{\circ} \mathrm{C}\right)$ than the pure polyester. This may be due to the more aggressive processing conditions (higher torque) that were needed to produce the blend. The higher torque would have induced a higher thermal degradation on PLA and, hence, a greater decrease on the molecular weight. However, the biggest difference lies in the onset of the coldcrystallization process, which occurs at $T_{\mathrm{c}}=89.2{ }^{\circ} \mathrm{C}$. This may indicate that chitosan acts as a nucleating agent, promoting a faster crystallization of PLA; however, the differences could be also associated with the higher decrease of the molecular weight of PLA during the processing of the blend. No significant changes are observed in the melting temperature between PLA and the blend with chitosan (155.3 and $155.7^{\circ} \mathrm{C}$, respectively), indicating that similar lamellae pop-
Table 3

Melting temperature and heat of fusion of the different samples analyzed by DSC, and the corresponding crystallinity degree within the synthetic polymer component

\begin{tabular}{lcll}
\hline Material & $T_{\mathrm{m}}\left({ }^{\circ} \mathrm{C}\right)$ & $\Delta H\left(\mathrm{Jg}^{-1}\right)$ & $X(\%)^{\mathrm{a}}$ \\
\hline PCL & 51.9 & 69.2 & 41.7 \\
25\%C+75\%PCL & 53.5 & 44.6 & 35.8 \\
50\%C+50\%PCL & 55.2 & 25.9 & 31.2 \\
PBS & 107.2 & 73 & 66.2 \\
25\%C+75\%PBS & 103.4 & 55.5 & 67.1 \\
$50 \% \mathrm{C}+50 \%$ PBS & 102.4 & 31.7 & 57.5 \\
$70 \% \mathrm{C}+30 \%$ PBS & 104.6 & 23.1 & 69.8 \\
PBSA & 83.7 & 40.2 & - \\
50\%C+50\%PBSA & 77.2 & 16.2 & - \\
\hline
\end{tabular}

${ }^{\text {a }}$ Crystallinity in the polyester fraction.

ulations are produced during cold crystallization in the two materials.

The DSC results on the PCL-based materials (Fig. 1b), in the region of the melting temperature of the polymer that include pure PCL, and blends with different chitosan contents (to study the effect of the polysaccharide) is summarized in Table 3. From the enthalpy values, the degree of crystallinity of the PCL component in the samples was calculated from the known PCL content and by assuming that the heat of fusion of completely crystalline PCL is $166 \mathrm{~J} \mathrm{~g}^{-1}$ [15]. The melting temperature tends to increase slightly with increasing chitosan content. In a separate study, an opposite behavior was observed for PCL/chitosan blends prepared by solvent casting [16]. The crystallinity of PCL tends to decrease with increasing chitosan content, similar to that observed in the work of Honma et al. [16]. This was attributed to hydrogen bond interactions between the carbonyl groups of the polyester and $-\mathrm{OH}$ and $-\mathrm{NH}_{2}$ groups in chitosan. Such interactions occur in the amorphous state, thus, suppressing the extent of crystallization of the PCL domains.

There is a general tendency for the depression of $T_{\mathrm{m}}$ with increasing chitosan content, except for the blend with $70 \%$ by weight of chitosan (Table 3). The degree of crystallinity of the PBS component was calculated from the known theoretical value of $\Delta H_{\mathrm{m}}$ for $100 \%$ crystalline PBS, that was estimated to be $110.3 \mathrm{~J} \mathrm{~g}^{-1}$, calculated on the basis of the group contribution method proposed by Van Krevelen [17]. In the PBS-based material there is a general tendency for the depression of $T_{\mathrm{m}}$ with increasing chitosan content, except for the blend with $70 \mathrm{wt} \%$ of chitosan (Table 3 ). The same trend was observed in blends of poly(3-hydroxybutyrate) and chitosan [18] and was attributed to strong intermolecular interactions between chitosan and the polyester chains, resulting in thinner lamellar thickness crystals. The chitosan/PBSA blends also exhibit a decrease in melting temperature (Table 3). The addition of $25 \%$ and $70 \%$ chitosan progressively increases the crystalline content of PBS, this behavior being the opposite of that observed for PCL. However, the introduction of $50 \%$ by weight of chitosan in PBS or in PBSA has a depressing effect on the degree of crystallinity of the polyesters. This 
may be an indication that the physical and thermal properties of such kinds of blends may not exhibit regular trends with the compositions, as the processing conditions may also have an important effect on the performance of the final materials.

\subsection{Mechanical properties}

The processing parameters for compounding and injection molding are summarized in Tables 1 and 2. It is well known that extrusion compounding parameters, such as screw profile, barrel temperature, and residence time, affect the morphology of blends. Similarly, several injection molding processing parameters, such as injection speed, packing pressure, barrel temperature, and mold temperature, may affect the tensile properties. In this study, no attempt was made to optimize properties. Rather, the conditions were selected for ease of processing and visual acceptance of molded parts.

Tensile strength, tensile modulus, and ultimate elongation are shown in Table 4. A typical stress-strain curve for the unfilled polyesters is shown in Fig. 2. The deformation is accompanied by the formation of a neck (except for PLA), where the true stress monotonically increases with the strain. Addition of chitosan led to a decrease in the tensile strength. This decrease is the smallest for PBTA and the largest for PBSA and PLA. Also, the level of decrease is insignificant for samples containing 25\% and 50\% chitosan in PBS. However, when the chitosan content is increased to $70 \%$, there is a further decrease in tensile strength from 28.7 to $21.3 \mathrm{MPa}$. The higher decrease in tensile strength with PLA is partly because PLA has been observed to be very sensitive to degradation during processing $[19,20]$. The presence of moisture has a particularly severe effect at higher temperatures because it induces hydrolysis. While PLA samples were dried prior to

Table 4

Tensile properties of various polyesters and their blends

\begin{tabular}{|c|c|c|c|}
\hline Materials & $\begin{array}{l}\text { Tensile strength } \\
\text { (MPa) }\end{array}$ & $\begin{array}{l}\text { Tensile } \\
\text { modulus (GPa) }\end{array}$ & $\begin{array}{l}\text { Elongation } \\
(\%)\end{array}$ \\
\hline PBS (1050) & $38.6 \pm 1.3$ & $0.611 \pm 0.01$ & $264 \pm 29$ \\
\hline PLA & $82 \pm 2.1$ & $2.4 \pm 0.13$ & $6.18 \pm 1.1$ \\
\hline PCL & $27.3 \pm 0.8$ & $0.378 \pm 0.016$ & $674 \pm 36$ \\
\hline PBTA & $20.2 \pm 0.3$ & $0.0817 \pm 0.0011$ & $1075 \pm 37$ \\
\hline PBSA (3001) & $41.4 \pm 1.1$ & $0.348 \pm 0.008$ & $311 \pm 20$ \\
\hline $\begin{array}{l}\text { PBS/Chitosan } \\
(75: 25)\end{array}$ & $28.7 \pm 0.8$ & $1.11 \pm 0.023$ & $6.4 \pm 0.6$ \\
\hline $\begin{array}{c}\text { PBS/Chitosan } \\
\text { (50:50) }\end{array}$ & $30.5 \pm 0.8$ & $1.98 \pm 0.03$ & $2.66 \pm 0.21$ \\
\hline $\begin{array}{l}\text { PBS/Chitosan } \\
\text { (30:70) }\end{array}$ & $21.3 \pm 1.6$ & $3.2 \pm 0.14$ & $1.37 \pm 0.036$ \\
\hline $\begin{array}{c}\text { PCL/Chitosan } \\
\text { (50:50) }\end{array}$ & $21.1 \pm 0.9$ & $1.84 \pm 0.069$ & $5 \pm 0.28$ \\
\hline $\begin{array}{l}\text { PBTA/Chitosan } \\
\quad(50: 50)\end{array}$ & $17.9 \pm 0.18$ & $0.722 \pm 0.024$ & $8.05 \pm 0.88$ \\
\hline $\begin{array}{c}\text { PLA/Chitosan } \\
(50: 50)\end{array}$ & $54.3 \pm 7.5$ & $3.74 \pm 0.19$ & $1.97 \pm 0.37$ \\
\hline $\begin{array}{l}\text { PBSA/Chitosan } \\
\quad(50: 50)\end{array}$ & $26.4 \pm 2.3$ & $1.74 \pm 0.041$ & $4.33 \pm 3.05$ \\
\hline
\end{tabular}

processing, the chitosan samples were used as received and had a moisture content of $6.9 \%$ on a dry weight basis.

The decrease in tensile strength is partially due to the thermodynamic immiscibility and inherent incompatibility between chitosan and polyester. The mechanical performance of a filled polymer depends on the strength and modulus of the filler. Yield strength of a heterogeneous polymer blend or composite is dependent on the yield strength of the matrix, the decrease of effective load bearing cross-section, and the interfacial adhesion between the matrix and the filler and its ability to transfer stresses across the interface [21]. Lack of adhesion led to the formation of pores due to the debonding of the fibers upon the application of stress in a particulate-filled material. Similar incompatibility has been observed between other natural polymers and polyesters [22-24].

Thus, because of the decrease in tensile properties upon the addition of chitosan, the blends used in this study can be characterized as having a reduced adhesive interaction between the chitosan and the polyesters. As the load is applied to the blend, the lack of interfacial adhesion between the chitosan and the polyester will limit the load transfer process. The lower the chitosan-matrix adhesion, the lower will be the stress at which debonding occurs, as the fibers are unable to reinforce the composite. There appears to be some adhesion between the two phases, as the tensile strength is unaffected when the chitosan content is increased from $25 \%$ to $50 \%$. The decrease in properties when the chitosan content is increased to $70 \%$ by weight can be attributed to inadequate mixing. It should also be noted that the plasticizer would have an adverse effect on the strength of the blends. At high chitosan content (70\%), the torque experienced was high enough that the die had to be removed when compounding. Also, at higher chitosan content, the

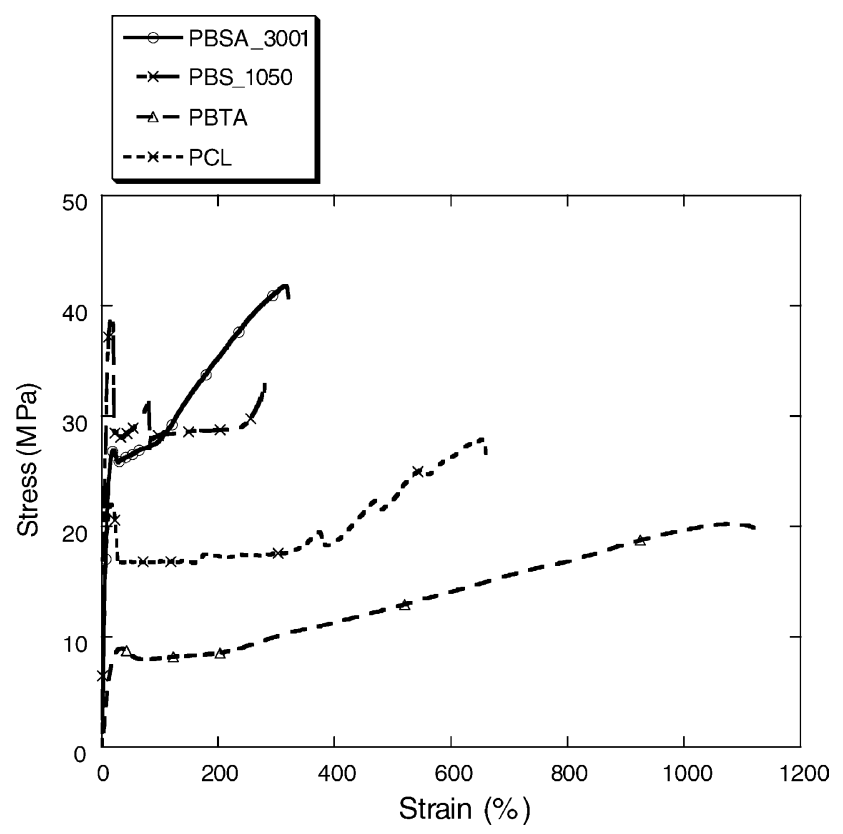

Fig. 2. Stress vs. strain curves for pure polyesters. 
filler inclusions form aggregates. These aggregates can lead to failure at lower stresses. Since the bonds between the filler particles are weaker than those between filler and the matrix, cavities would be formed, resulting in failure at lower stresses.

The shape of the stress-strain curve is affected by the addition of chitosan. For pure polymer (except for PLA), the behavior is similar to that of ductile polymers (Fig. 2). PLA is the only polymer with a glass transition greater than room temperature and, hence, displayed brittle failure. PBSA, PBTA, and PCL all show strain hardening. In the case of PBS, tensile stress showed an upper yield point followed by a drop in stress due to a reduction in the cross-sectional area due to necking. After yielding, the stress dropped and remained at a constant value for the duration of cold drawing (necking extension). The deformation mechanism of polymer filled with particles depends on the relationship between the strength and the yield stress of the unfilled polymer [25]. If the strength of the unfilled polymer is lower than its yield stress, an increase in particle content leads to an embrittlement of the polymer. This would appear to be the case for blends of chitosan and PBS. For both 50\% chitosan and PBS and 50\% chitosan and PLA, failure occurs at low strains, and the material fails immediately after stress reaches maximum.

The elongation of break shows a marked decrease upon the addition of chitosan (Table 4). There is approximately a two order of magnitude decrease in the elongation upon the addition of $25 \%$ chitosan. However, the elongation is less affected as the chitosan content is increased from $25 \%$ to $70 \%$. According to Dubnikova et al. [26], there is a critical filler volume fraction $(\phi)$ below which the samples deform by necking. Beyond this critical value, there is negligible shrinkage of the cross-sectional area. For aluminum hydroxide filled PP, the authors observed for certain filler dimensions that elongations were independent of filler content $\phi>0.2$. The elongations at failure for all blend compositions are of the same order of magnitude. While the pure polyesters underwent plastic deformation, the blends with 50\% PBS displayed brittle failure, and those with 50\% PBTA, 50\% PBSA, 50\% PCL and 75\% PBS had uniform yield (Fig. 3). Chitosan/PLA blends displayed brittle failure. The deformation and failure of the composites occurred without any shrinkage of cross-sectional area, including those with uniform yield.

The modulus increased with increased chitosan content (Table 4). This suggests that there is sufficient stress transfer across the polyester-chitosan interface. The chitosan filler in the matrix restrains the movement of the polyester phase near the vicinity of each particle, contributing to an increase in the modulus. The increase in modulus was the highest for blends with PBTA and lowest for blends containing PLA. When a relatively softer matrix is reinforced by a high modulus filler, the polymer adjacent to the filler particle is highly restrained; this enables a major portion of the load to be carried by the filler.

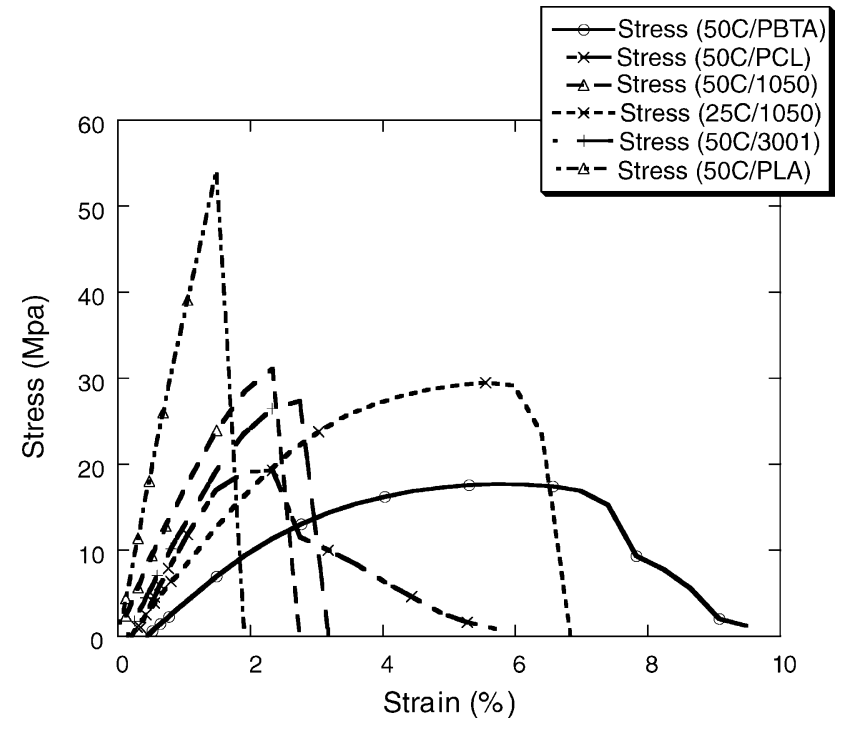

Fig. 3. Stress vs. strain curves for blends of polyester and chitosan.

\subsection{Optical microscopy}

It is well known that processing conditions affect the morphology of the molded part. Furthermore, in this study, samples of different compositions could not be molded under the same molding conditions. The processing of the materials, included a blending stage in a twin-screw extruder, was followed by grinding and subsequent injection moulding of test bars. Optical microscopy of injection molded samples is shown in Figs. 4 and 5. The staining with eosin was effective in identifying the chitosan domains in the blends. It appears that there is an adequate mixing of the polyester/chitosan blends. The optical microscopy micrographs were obtained both at the surface of injection molded tensile bar specimens (Fig. 4) and on cross-sections of cryogenically-fractured samples (Fig. 5). The morphology of the molded samples was observed to be heterogeneous and displayed a

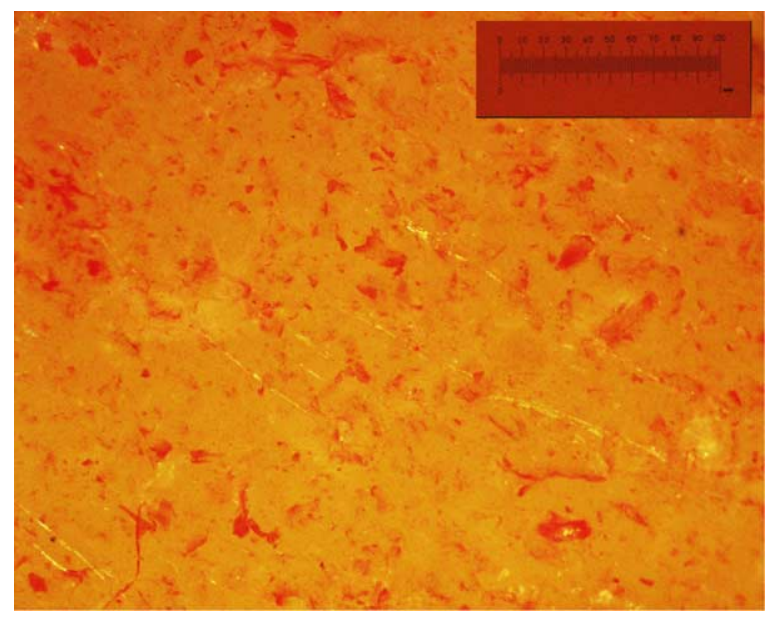

Fig. 4. Optical micrograph of the surface of injection molded tensile bar of blends containing $50 \%$ chitosan and $50 \%$ PBSA. 


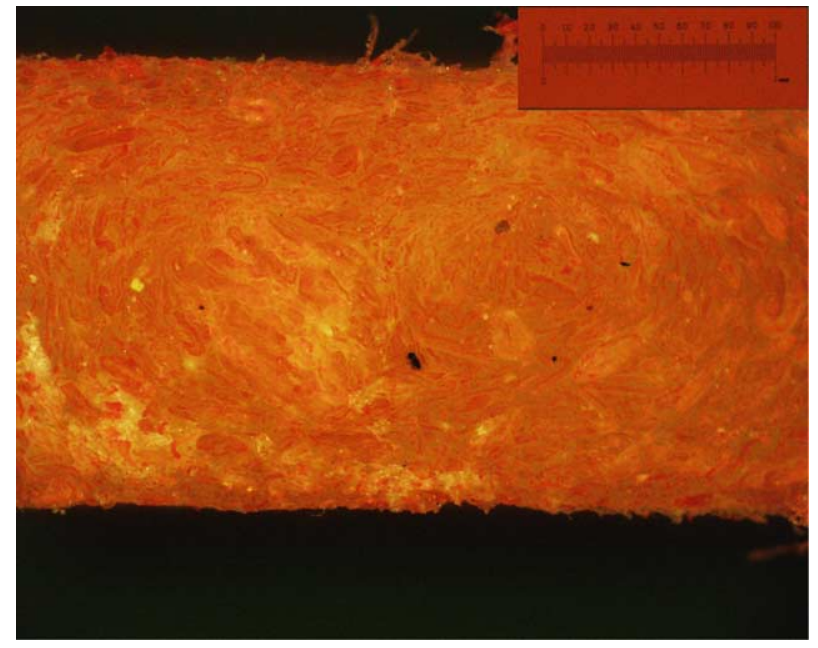

Fig. 5. Optical micrograph of cryogenically-fractured cross-section of blends containing $25 \%$ chitosan and $75 \%$ PBS

skin-core distribution. The skin is composed almost entirely of polyester. Located below this polyester skin is the chitosan dispersed in the polyester, as is clearly observed in Fig. 5.

In all the cases examined, including the high chitosan (70\%) containing blends, it is observed that chitosan remained dispersed in a continuous phase of polyester. This is expected since the chitosan particles do not melt. As the chitosan contents increased in the blends from $25 \%$ up to $70 \%$, there is not a proportional morphology evolution in the images of injection molded surfaces. The observation of the cross-sections show a much clearer correlation between chitosan content and the area stained red (chitosan) in the images. This is an indication that the chitosan domains tend to be enclosed in the inner regions of moldings and confirms the earlier observation that the continuous phase consists of polyester and the dispersed phase is composed of chitosan domains. The distribution of chitosan domains in the cross-section images is controlled by the flow pattern. A circumferential and symmetric alignment of the domains with respect to the central point of the cross-section and defining a skin/core structure characteristic of injection mouldings is observed. In the blends with higher chitosan (70\%), the distinct skin/core structure and the symmetric alignment is less visible, probably because of agglomeration of chitosan domains leading to chitosan-rich clusters.

Though difficulties were experienced in using optimal extruder configuration (reverse mixing element and use of die) due to high torque and viscous dissipation, an experiment was conducted to extrude blends of $50 \%$ chitosan and $50 \%$ PBSA using a reverse mixing element and a die. Optical micrographs of this blend indicate that the chitosan is much more distributed, as observed by the stained image of the chitosan domains (figure not shown). The mixing element in the extruder seems to enhance the quality of the mixing, however, it also causes a large increase in the torque required to process the materials, hindering its use in all other blends. The use of this reverse mixing element in the screw seems to be more effective in the dispersive mixing than in the distributive mixing.

\subsection{Scanning electron microscopy}

Injection molded and extruded samples etched with acetic acid and observed under SEM also confirm the findings from optical microscopy that the chitosan is located preferentially under the skin. Of the components involved in this study, chitosan dissolves in dilute acetic acid, while the polyesters (except PLA) were relatively stable in acetic acid. The observations of the surface and of cross-section images indicate that the chitosan domains are 100-300 $\mu \mathrm{m}$ long with thicknesses in the order of $15-30 \mu \mathrm{m}$. It has been reported [27] that chitosan exists in the form of fibers that have thickness of around $120 \AA$. SEM micrograph of chitosan powders (as received from the manufacturer and used in the blends without any modification) indicates that the thickness of the particles is much greater than that reported by Cartier et al. [27]. This would indicate that chitosan fibers are agglomerated into thicker particles prior to processing, giving the appearance of sheath-like structure. Due to insufficient torque during processing, these particles are unable to completely disperse into individual fibers. It is possible to observe some variation of the chitosan domain size, most probably because of local variation of mechanical energy dissipation during processing. The clustered chitosan fibers are bent and appear to be compliant enough to adapt their geometry to the stresses experienced during processing, without breaking.

The SEM of injection molded specimens after tensile failure when stretched in the machine direction of chitosan/PBS blends as a function of chitosan content are shown in Fig. 6. Agglomerated chitosan fibers under SEM are present in the form of thin sheath-like structures which have dimensions in the $10-20 \mu \mathrm{m}$ range or agglomerated ellipsoidal particles with dimensions in the 100-150 $\mu \mathrm{m}$ range. The thickness of the chitosan sheath varied with the composition; the higher the chitosan content, the thicker the sheath. We were unable to etch samples containing $70 \%$ chitosan by weight as they collapsed. The formation of sheaths (Fig. 6b) is an indication of the bundling of agglomerated chitosan fibers.

In general, chitosan domains of oblong shape are dispersed in the polyester matrix. The macroscopic deformation initially localized at the weakest point in the specimen. Debonding between the dispersed chitosan particles and the polyester matrix is apparent in the SEM images because of the lack of adhesion. This lack of adhesion results in interfacial slippage between the chitosan and the polyester matrix, leading to voids and cavitations. The holes resulting from the particle pull-out from the matrix is evident in most of the compositions (except in the case of chitosan/PLA). In addition to holes, thin wedges are also apparent in the matrix (Fig. 6c). These wedges are a result of agglomerated chitosan fibers removed from the matrix. In some blends, these agglomerated fibers stack up, leading to the formation of sheaths (Fig. 6b). This gives rise to cluster-like morphology of the 

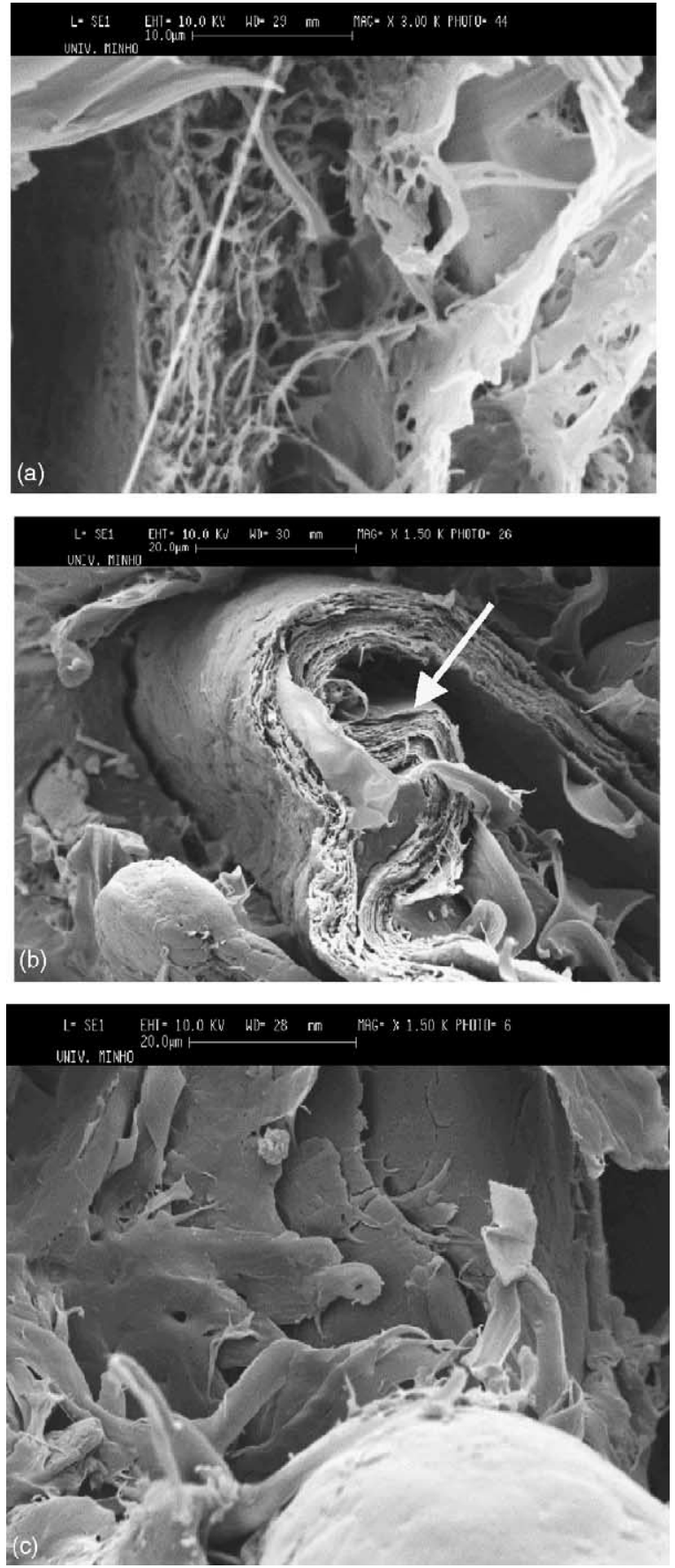

Fig. 6. Scanning electron micrographs of tensile surfaces of chitosan and PBS (a) 25\% chitosan; (b) 50\% chitosan (the arrow denotes the sheath of chitosan); and (c) $70 \%$ chitosan.

dispersed phase. The stresses generated during the processing were unable to destroy these structures. The formation of these sheaths decreases the aspect ratio of the fillers and can lead to significant decreases in reinforcement efficiency [28-30].

The tensile-fractured surface of $25 \%$ chitosan $/ 75 \%$ PBS and $50 \%$ chitosan $/ 50 \%$ PBSA shows the formation of fine fibrous structure (Fig. 6a). The polyester domains are elongated into fine fibrils of submicron dimensions in the core layers of the blend. Fibrous structures were also observed for 50\% chitosan blended with PBTA (Fig. 7a) and PCL, though the diameters of the fibers were considerably larger (of micron scale) than those observed in blends containing PBS and PBSA. This would indicate that there is localized micronecking and fibrillation even in blends containing as high as $50 \%$ by weight of chitosan.

For chitosan/PLA blends (figure not shown), the upper and lower surfaces appeared smooth, with little evidence of any protrusion or loose chitosan particles. The number of cavities in chitosan/PLA is also sharply lower, as well as of smaller diameter, than those observed in blends of other polyester with similar chitosan content. This is due to the mode of failure where the chitosan particle undergoes minimal deformation. For the more ductile polymers (PCL, PBTA and PBSA), the fibrous appearance of the surface is due to the stretching of the surrounding polymer threads and their subsequent failure. As the chitosan content is increased in chitosan/PBS blends, the surface becomes smoother, indicating a decline in ductility.

It is also evident that the number of cavities in the cross-section of the extruded samples (figure not shown) are much lower than those in the tensile-fractured surface. The cryogenically-fractured tensile bar surfaces for most blends are relatively smooth compared to those tensile bar surfaces subjected to tensile fracture (Fig. $7 \mathrm{a}$ and b). For
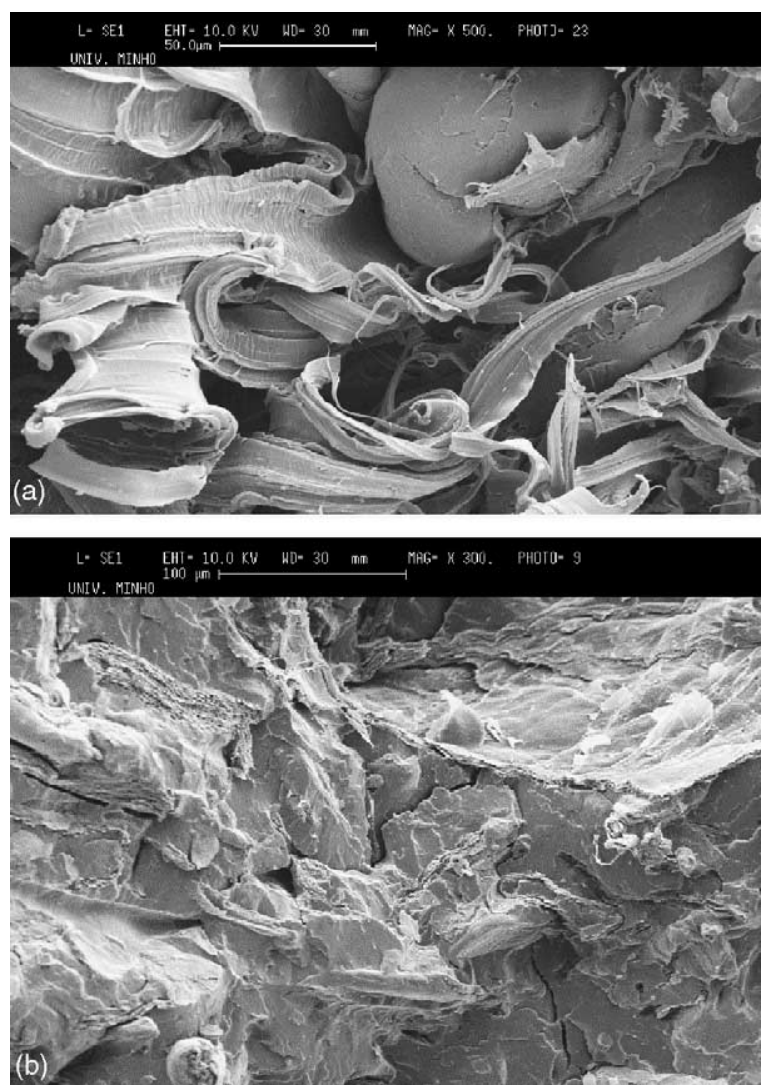

Fig. 7. Blends of 50\% chitosan and 50\% PBTA (a) tensile-fractured surface and (b) cryogenically-fractured surface. 
chitosan/PLA-fractured surfaces (figure not shown), it is observed that stacks of chitosan sheaths, inadequately dispersed, have induced local breakage. Cavities were also observed in the extruded specimens of the blends. The cavities that exist in extruded samples are probably due to foaming as a result of the presence of moisture.

The cavities showed both spherical and ellipsoidal form and had a size distribution. In addition, cavities of differing sheath thickness are also observed. The cavitation is more apparent in the blend containing $25 \%$ chitosan than in the blend containing $70 \%$ chitosan (Fig. 6a versus Fig. 6c). This difference is due to the mode of failure of the two samples. The $70 \%$ chitosan-containing blend fractures in the brittle mode, where there is very little deformation. The $25 \%$ chitosan-containing blend has a higher elongation (Fig. 6a). The blend containing 50\% chitosan, as expected, shows an intermediate behavior.

The surfaces of some of the cavities are relatively smooth, indicating a lack of complete adhesion. Also, there appeared to be a lack of wetting between the chitosan fiber and the polyester. Bundles of sheaths were also observed in the samples (Fig. 7b), indicating local domains where mixing was insufficient. The diameter of the bundles increased as the concentration of chitosan increased. This is attributed to fiber agglomeration. The effect is similar to that reported for fiber reinforced polymers. The packing density of randomly oriented fiber is low. When the volume fraction of fiber being compounded is greater than its natural packing density, the fiber must either break into shorter fragments or form bundles to conform to space filling requirements [31]. Fiber breakage can be achieved by increasing the pressure. However, we were limited by maximum amperage of the extruder motor. The addition of as little as $25 \%$ chitosan increased torque significantly over what was required to process the synthetic polyester. This necessitated a decrease in screw speed (hence pressure) as well as adjusting screw profile (such as no reverse element) and the removal of the die to further decrease the pressure.

To identify the dispersion of chitosan domains in the blend matrix, a set of samples were etched by immersing in an acetic acid solution and observed under SEM. Chitosan is soluble in $1 \%$ acetic acid solution. A cryogenically-fractured surface of $50 \%$ chitosan and PCL with and without etching is shown in Fig. 8. The cavities observed in these etched samples are those of chitosan. The particle size of chitosan ranges from $15-145 \mu \mathrm{m}$. A range of cavity dimensions dispersed randomly in the etched samples was observed. The shapes of these cavities range from spherical to those resembling stacked sheaths. The dimensions and shapes of the holes correspond to those observed for unprocessed chitosan powder under SEM. It is also possible that there is some agglomeration of chitosan particles in the blends, leading to hole dimensions larger than those observed for unprocessed chitosan powder under SEM. The cavities are not normal to the fracture surfaces, indicating that the chitosan agglomerates do not necessarily align themselves in the direction of flow.
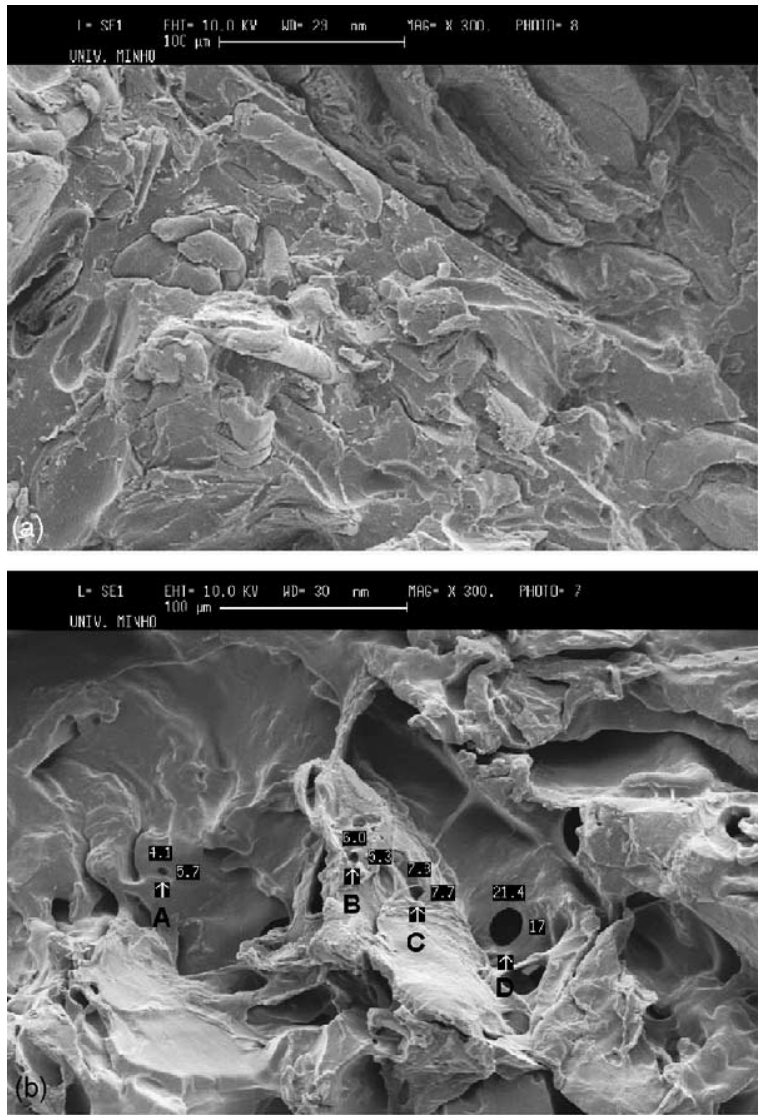

Fig. 8. Blends of 50\% chitosan and 50\% PCL: (a) Cryogenically fractured and (b) cryogenically fractured and etched in $1 \%$ aqueous acetic acid for $3 \mathrm{~h}$ (A: $4.1 \times 5.7 \mu \mathrm{m}$; B: $6.0 \times 5.3 \mu \mathrm{m}$; C: $7.8 \times 7.7 \mu \mathrm{m}$; D: $21.5 \times 17.0 \mu \mathrm{m})$.

\subsection{Small-angle X-ray scattering}

Further insights on the microstructure at the lamellae scale can be obtained from SAXS. Selected 2D SAXS patterns of injection molded parts are shown in Fig. 9. All materials exhibited the typical scattering features associated with the electron density contrast between the alternating crystalline and amorphous layers. PCL displays a noticeable orientation of its lamellar morphology in the direction of the melt flow inside the mold. The inclusion of $25 \%$ and $50 \%$ by weight of chitosan seems not to affect this orientation; only the intensity of the diffraction pattern decreases, as expected. PBSA samples also exhibit a strong anisotropy, also in the direction of the melt flow. However, the lamellar structure, although maintaining this orientation, is strongly depressed with the inclusion of $50 \%$ weight of chitosan. The 2D SAXS patterns of the PBS parts do not exhibit the anisotropy found in the other materials. It should also be mentioned that despite the relative high degree of crystallinity of PBS (relative, for example, to PCL), the intensity of the SAXS pattern is quite low, probably indicating that the lamellar organization is not as efficient as in PCL.

The 2D patterns were integrated (using the X-ray software, version 1.0) to estimate the intensity as a function of 


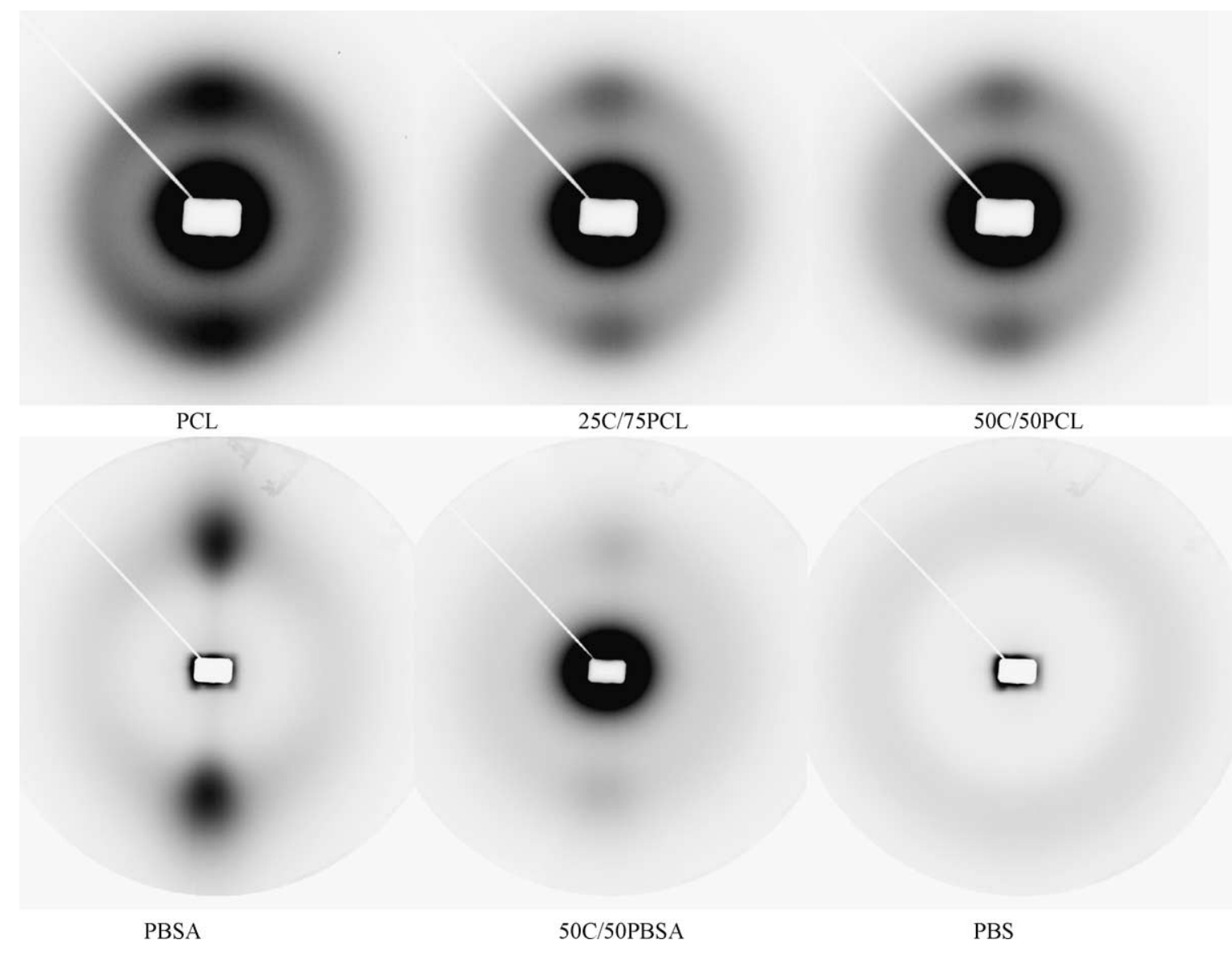

Fig. 9. 2D-SAXS patterns of injection-moulded samples.

the scattering vector, $s$. Lorentz-corrected scattering profiles analyzed for some materials is shown in Fig. 10. In the low angular regions, a typical increase of the intensity is detected, which, due to the low signal, indicated that no regular heterogeneous structures are present with dimensions larger than the crystalline and amorphous layers.

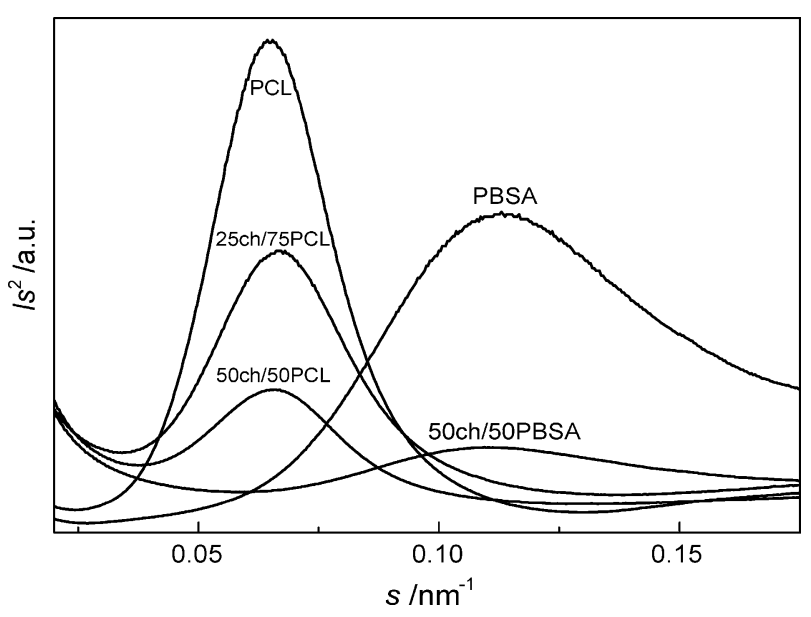

Fig. 10. Lorentz-corrected SAXS profiles of some PCL- and PBSA-based samples.
As expected, the intensity of the main diffraction peaks decreased strongly with the incorporation of chitosan, as the total crystallinity (and, thus, the development of crystalline lamellae) is depressed. The position of the peaks was used to calculate the average long period, $L=1 / s_{\max }$. For the PCL-based materials, we found that $L$ ranged between 14.9 and $15.3 \mathrm{~nm}$, with no particular trend with the chitosan content. Also for the PBSA and 50\% chitosan and 50\% PBSA materials, a similar long period was found $(8.8$ and $9.0 \mathrm{~nm}$, respectively) for the lamellar structure of the polyester. From the results of the PBS system, we conclude that the average long period is always lower here than in PBSA, being near $7.5 \mathrm{~nm}$, independently of the chitosan content. The results shown in Fig. 10 clearly indicate that the addition of the polysaccharide on the studied polyesters does not significantly affect the morphology developed at the lamellar scale.

\subsection{Wide-angle $X$-ray scattering}

Representative WAXS patterns on some selected PCLand PBS-based materials are shown in Fig. 11. In the first case, two main diffractions, at $2 \theta$ around $21^{\circ}$ and $23^{\circ}$, attributed to the (1 10$)$ and (2 00 ) planes in PCL are detected [32]. Neither peak changes their position upon blending with chitosan. Moreover, the half-width of both peaks are 


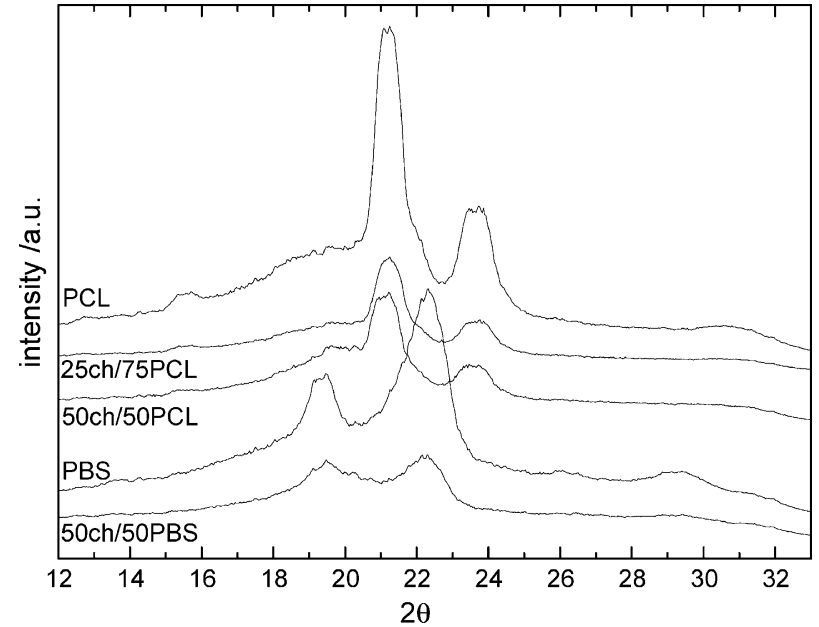

Fig. 11. WAXS patterns of some PCL- and PBS-based samples, where the effect of the addition of chitosan is investigated.

maintained, indicating that the PCL crystal sizes are also maintained when chitosan is added. It is interesting to compare these results with those obtained by Honma et al. [16], where blends of PCL with chitin and chitosan were prepared by solvent casting. For solvent cast blends, they observed differences in the interplanar spacings of the $\left(\begin{array}{lll}1 & 1 & 0\end{array}\right)$ planes with the inclusion of the polysaccharides, the $b$ axes of the PCL orthorhombic unit cell being more spread out in the blends. However, no differences were found in the spaces of the $(200)$ planes [16]. It can therefore be concluded that considerable molecular interaction occurs between PCL and chitosan when the blend is processed by solvent-casting, influencing the crystalline development of PCL. By contrast, in the PCL blends prepared by injection molding, it seems that these interactions are weaker, as no influence in the crystalline morphology is detected in either the crystal unit (probed by WAXS) or lamellar (probed by SAXS) length scales.

The diffraction diagrams for the PBS-based injection molded parts (Fig. 11) display a peak at $2 \theta \approx 19.3^{\circ}$, assigned to the (11-1) and (l0 02 ) planes, a peak of $\left(\begin{array}{lll}1 & 1 & 0\end{array}\right)$ at $2 \theta \approx 22.2^{\circ}$, with a shoulder at lower angles (better defined in the blends and composites) associated with the (0 12 ) planes, a peak of $(12-1)$ at $2 \theta \approx 25.9^{\circ}$ and a peak of (1 111$)$ at $2 \theta \approx 29^{\circ}$. An electron diffraction study showed that PBS chains crystallize in monoclinic crystal lattice, with $a=0.523 \mathrm{~nm}, b=0.908 \mathrm{~nm}$, $c=1.079 \mathrm{~nm}$, and $\beta=123.87^{\circ}$ [33]. It can be seen in Fig. 11 that the position of the diffraction peaks is not altered with the introduction of chitosan, as observed previously for the PCL system. Only the intensity of the peaks is altered due to the loss of total crystallinity (with the blending with chitosan).

\section{Conclusions}

Chitosan, an important biomaterial, can be melt blended with several biodegradable polyesters to produce materials with a range of acceptable properties. Addition of chitosan to PLA decreased the glass transition temperature as well as the temperature of the onset of crystallization. For chitosan/PCL blends, the degree of crystallinity decreased with increased chitosan content. When the chitosan content in PBS increased, the melting temperature was found to decrease. For blends containing $25 \%$ and $70 \%$ chitosan, the crystallinity of the PBS increased, while at the intermediate chitosan content $(50 \%)$, it decreased.

The tensile strength decreased with increased chitosan content while the modulus increased. A small amount of chitosan content caused a significant decrease in the elongation. No necking was observed in the filled polymers. These blends displayed a skin core morphology. Chitosan domains tend to be enclosed in the inner regions of moldings, confirming the observation that the continuous phase consists of polyester and the dispersed phase is composed of chitosan domains. In all the blend compositions, the distribution of chitosan domains in the cross-section images seems to be controlled by the flow pattern, leading to a nearly radial and symmetric distribution of the domains with respect to the central point of the cross-section. Dispersed chitosan particles agglomerate to sheaths as the chitosan content is increased. The size distribution of chitosan observed in the blends is consistent with the range of sizes observed for pure chitosan under SEM. Qualitatively, the average particle size seems to decrease after processing, particularly in the $25 \%$ chitosan blends. This is probably due to the stresses experienced during extrusion, milling and subsequent injection moulding. There is some debonding between the chitosan platelets and the polyester matrix, as evident in the SEM of the tensile-fractured surfaces. Platelet pull-out results in holes in most of the matrices. The only exception is chitosan/PLA blends whose crosssection showed little evidence of any protrusion or loose chitosan particles. As the chitosan content is increased in chitosan/PBS blends, the fracture surface becomes smoother, indicating a decrease in ductility. Blends of chitosan with PCL, PBTA, or PBSA display fibrous appearance at the fractured surface due to the stretching of the polymer threads.

When cryogenically-fractured surfaces are etched with acetic acid to remove chitosan, cavities corresponding to dimensions of chitosan platelets are evident. These cavities have smooth surfaces, indicating that the chitosan fibers do not completely adhere to the polyester matrix. Some cryogenically-fractured surfaces of blends show fibrillar polymer structure.

These polyesters display SAXS patterns that are consistent with a typically lamellar morphology. The lamellar orientation of the polyesters (PCL, PBSA) are unaffected by the addition of chitosan. However, the addition of chitosan does suppress the lamellar development. PBS, on the other hand, while crystalline, displays a more isotropic orientation. The intensity of the main diffraction peaks decreased with the incorporation of chitosan, as the total crystallinity is reduced. The two main diffraction peaks in PCL, observed using WAXS, are unaffected by the addition of chitosan. Similarly, the inclusion of HA does not influence the position of 
the diffraction peaks of PCL. The X-ray diffraction results confirm the morphological observations that the chitosan and the polyester are phase-separated systems.

\section{Acknowledgement}

L.F. Boesel acknowledges Fundação Coordenação de Aperfeiçoamento de Pessoal do Ensino Superior (CAPESBrasília, Brazil) for the Ph.D. grant. M.B. would like to thank FLAD (Fundação Luso-Americana para o Desenvolvimento) for generous support of sabbatical funds towards this research. Financial support for this work was also provided by FCT through the POCTI and FEDER programs and EU Integrated Project Genostem (Adult Mesenchymal Stem Cells Engineering for connective tissue disorders: from the bench to the bed side).

\section{References}

[1] B.S. Kim, D.J. Mooney, Trends Biotechnol. 16 (5) (1998) 224-230.

[2] I. Engleberg, J. Kohn, Biomaterials 12 (1991) 292-304.

[3] R.A.A. Muzzarelli, Enzymatic synthesis of chitin and chitosan, in: Chitin, Pergamon Press, Oxford, 1977, pp. 5-17.

[4] T. Chandy, C.P. Sharma, Biomater. Artif. Cells Organs. 18 (1990) $1-24$.

[5] M.F.A. Goosen, Appplications of Chitin and Chitosan, Technomic Publishing Co. Inc., Pennsylvania, 1997.

[6] D.K. Singh, A.R. Roy, J. Appl. Polym. Sci. 66 (1997) 869-877.

[7] D.K. Singh, A.R. Roy, J. Appl. Polym. Sci. 53 (1994) 11151121.

[8] H.S. Blair, J. Guthrie, T. Law, P. Turkington, J. Appl. Polym. Sci. 33 (1987) 641-656.
[9] A. Lagos, J. Reyes, J. Polym. Sci. Part A: Polym. Chem. 26 (1988) 985-991.

[10] Y. Shigeno, K. Kondo, K. Takemoto, J. Macromol. Sci. Chem. A17 (1982) 571-583.

[11] S. Yoshikawa, T. Takeshi, N. Tsubokawa, J. Appl. Polym. Sci. 68 (1998) 1883-1889.

[12] P. Gong, L. Zhang, L. Zhuang, J. Lu, J. Appl. Polym. Sci. 68 (1998) $1321-1329$.

[13] R.A.A. Muzzarelli, P. Ilari, M.B. Tomasetti, J. Biomater. Sci. Polym. Ed. 6 (1994) 541-547.

[14] T. Chandy, C.P. Sharma, J. Appl. Polym. Sci. 44 (1992) 2145-2156.

[15] H.L. Chen, L.J. Li, T.L. Lin, Macromolecules 31 (1998) 2255-2264.

[16] T. Honma, T. Senda, Y. Inoue, Polym. Int. 52 (2003) 1839-1846.

[17] D.W. Van Krevelen, Properties of Polymers, Elsevier, Amsterdam, 1990.

[18] M.K. Cheung, K.P.Y. Wan, P.H. Yu, J. Appl. Polym. Sci. 86 (2002) 1253-1258.

[19] D.H.R. Ramkumar, M. Bhattacharya, Polym. Eng. Sci. 38 (1998) 1426-1435.

[20] K. Jamshidi, S.H. Hyon, Y. Ikada, Polymer 29 (1988) 2229-2234.

[21] B. Pukanszky, F.H.J. Maurer, J.W. Boode, Polym. Eng. Sci. 35 (1995).

[22] R. Mani, M. Bhattacharya, Eur. Polym. J. 37 (2001) 515-526

[23] J. Jacob, M. Bhattacharya, Polym. Int. 48 (1999) 1165-1172.

[24] U.R. Vaidya, M. Bhattacharya, J. Appl. Polym. Sci. 52 (1994) 617-628.

[25] S. Bazhenov, Polym. Eng. Sci. 35 (1995) 813-822.

[26] I.L. Dubnikova, V.G. Oshmyan, A.Ya. Greenberg, J. Mater. Sci. 32 (1997) 1613-1622.

[27] N. Cartier, A. Domard, H. Chanzy, Int. J. Biol. Macromol. 12 (1990) 289-294.

[28] T.D. Fornes, D.R. Paul, Polymer 44 (2003) 4993-5013.

[29] B.E. Clements, E.M. Mas, J. Appl. Phys. 90 (11) (2001) 5522-5534.

[30] E.M. Mas, B.E. Clements, J. Appl. Phys. 90 (11) (2001) 5535-5541.

[31] K.E. Evans, A.G. Gibson, Composites Sci. Tech. 25 (1986) 149-162.

[32] Y. Chatani, Y. Okita, H. Yadokoro, Y. Yamashita, Polym. J. 1 (1970) 555.

[33] K.J. Ihn, E.S. Yoo, S.S. Inn, Macromolecules 28 (1995) 2460-2464. 